\title{
Controlling Visually Induced Self-Motion Perception: Effect of Overlapping Dynamic Visual Noise
}

\author{
Hiroyuki Ito and Hiroshi Takano \\ Department of Visual Communication Design, Kyushu University
}

\begin{abstract}
The effect of overlapping dynamic visual noise on visually induced self-motion perception (vection) by upward or downward optical flow was tested. The dynamic visual noise consisted of rapidly refreshed sparse random dots. Binocular disparity of the overlapping noise plane was varied. The results showed that when the noise was presented on the flow plane or on a plane farther than the flow plane, vection was totally impaired. This demonstrates that dynamic visual noise is functionally equivalent to static patterns in the vection suppression effect. A possibility of dynamic visual noise as a vection suppressor in an application on a 3-D display is discussed in relation to simulator sickness. $J$ Physiol Anthropol Appl Human Sci 23(6): 307-311, 2004 http://www.jstage.jst.go.jp/browse/jpa
\end{abstract}

Keywords: self-motion perception, vection, binocular disparity, optical flow, 3-D, simulator sickness

\section{Introduction}

When one observes visual motion covering a large region in the visual field, self-motion in the direction opposite to that of the visual motion is sometimes induced. This kind of induced self-motion perception is called "vection". Induced selftranslation perception has been investigated by using linear motion of dots on a flat screen (Johansson, 1977; Kano, 1991; Nakamura and Shimojo, 1999; Ito and Shibao, 1999; Ito, 2004) or by using expanding flow patterns that induce forward vection (Andersen and Braunstein, 1985; Palmisano, 1996). The present study investigates a factor that suppresses vection.

Ohmi et al. (1987) and Ohmi and Howard (1988) showed the importance of the farther stationary stimulus on suppressing vection from rotating or expanding flow. The first author and colleagues also showed the importance of the farther flow in determining a basic vection direction using translational and rotational flows (Ito and Fujimoto, 2003), and expanding and contracting flows (Ito and Shibata, in press). In any case, vection is essentially dominated by the farther visual stimulus.

To correctly understand why a farther static stimulus suppresses vection, we tested the suppression effect by superimposing dynamic visual noise on a motion display and compared it with the suppression effect of a static stimulus. Here we investigate which condition is essential for the vection suppression by the farther stimulus: a static condition or a condition that indicates no consistent motion direction. The farther static stimulus can be a reference frame indicating that there is no relative motion between the observer and the background, whereas dynamic visual noise does not constitute a steady reference frame. If the difference were critical, only the static stimulus may suppress vection. On the other hand, both stimuli do not suggest an observer's self-motion when they are presented alone, because one is static and the other presents random motion. Therefore, it is also possible that presenting these stimuli on the farther plane would make them dominant in determining vection and suppress vection, as a result.

\section{Methods}

Four subjects including the authors participated in the experiment. All subjects had normal or corrected-to-normal vision.

The stimulus display was projected on the screen of a rear projection system (Electrohome Electronics, DRAPAR). The size of the screen was $138 \mathrm{~cm}$ (horizontal) $\times 104 \mathrm{~cm}$ (vertical). The viewing distance was $90 \mathrm{~cm}$. The screen subtended visual angles of $75 \mathrm{deg}$ (horizontally) and $60 \mathrm{deg}$ (vertically). Black cloth covered the left, right and upper sides of the subjects, so that they could not see anything but the display. The stimulus display was generated by a computer (SHARP CZ-644C). The display for each eye was treated as a 256 (vertical) $\times 512$ (horizontal) dot matrix. The resolution was not so high, but the quality of motion display was enough to compel subjects to feel self-motion. The dot positions that created motion impression were refreshed at $55 \mathrm{~Hz}$ while images on the screen were refreshed at $110 \mathrm{~Hz}$, with image-presentation alternating between each eye. Subjects wore a LCD shutter goggle (CrystalEYES2) to achieve stereoscopic view. The number of dots on each flow pattern (upward or downward) and the 


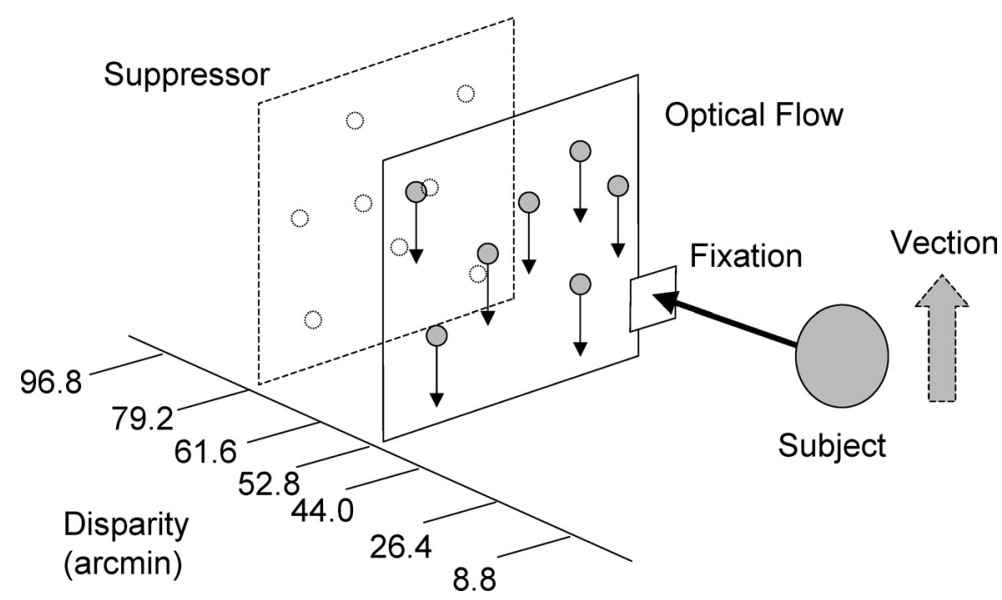

Fig. 1 Simulated depth planes. Upward or downward flow was presented on a fixed depth plane perceptually farther than the fixation square. The vection suppressor was static or dynamic random dots presented on a depth plane chosen from seven possible disparity planes.

suppressing pattern was 400 for all conditions, i.e., when upward flow and dynamic visual noise were presented at the same time, there were 800 dots on the screen for each eye. The luminance of the dot and the background was $7.0 \mathrm{~cd} / \mathrm{m}^{2}$ and $0.01 \mathrm{~cd} / \mathrm{m}^{2}$, respectively, when measured through the goggle. The diameter of each dot was $8.8 \mathrm{~min}$ of arc in visual angle.

The flow conditions used in the experiment are shown in Fig. 1. Two directions of flow were used: one was upward motion, to induce downward vection, and the other was downward motion, to induce upward vection. Each of these patterns was strong enough to produce vection when it was presented alone, as shown in the results. The dot speed was $20.6 \mathrm{deg} / \mathrm{s}$ in both the upward or downward direction. The dots that disappeared at the edge of the screen reappeared at the opposite edge, i.e., the motion pattern was endless. Each flow was presented on a disparity plane that was constant at 52.8 arcmin in an uncrossed direction.

There were two vection-suppressing stimuli. One consisted of 400 static dots positioned randomly over the screen. The other was dynamic visual noise consisting of 400 twinkling dots, i.e., changing their positions randomly synchronized with each screen-refreshment. The disparity of each suppressing stimulus was varied in 7 steps $(8.8-96.8 \mathrm{arcmin}$ in an uncrossed direction).

Using a chinrest, subjects viewed a square at the center of the screen (zero disparity plane). The size of the square was approximately $8.5 \mathrm{deg}$ in the horizontal and vertical dimensions. The motion displays were presented for one minute as one trial. Subjects continued to press one mouse button during periods of weak vection and pressed two buttons when they experienced strong vection. When they did not feel any vection, they released both buttons. The button condition was scanned at $27.5 \mathrm{~Hz}$. Trials under all conditions were given to the subjects in a random order with 5 repetitions (at least) of each trial. The repeatedly measured data were averaged within subjects.

\section{Results}

As shown in Fig. 2, when the static dots were presented on a larger-disparity plane (i.e., a plane that was perceptually farther than the flow plane), vection duration was drastically shorter than when they were presented on a smaller-disparity plane. The dynamic visual noise clearly had the same effect as that of the static dots.

A three-way repeated measures ANOVA revealed that only the main effect of disparity was significant $(F(6,84)=28.46$, $p<.0001$ ). The main effects of the flow direction (upward or downward) $(F(1,84)=1.44, p>.05)$ and the quality of the suppressor (static or dynamic dots) $(F(1,84)=.12, p>.05$ ) were not significant. No interaction was significant $(p>.05)$. LSD tests revealed that there were significant differences between the 8.8 arcmin and all other disparity conditions but the 26.4 arcmin condition $(p<.05)$, between the $26.4 \mathrm{arcmin}$ condition and all other disparity conditions but the $8.8 \mathrm{arcmin}$ condition, and between the 44 arcmin condition and all other disparity conditions. The difference between the 52.8 and 96.8 arcmin conditions was also significant. Differences between the other combinations of the disparity conditions were not significant $(p>.05)$.

According to the number of the pushed button (i.e., 0 for no vection, 1 for weak vection and 2 for strong vection), vection intensity was evaluated and averaged across one minute of each trial. Figure 3 shows the average intensity. The tendency is the same for the duration results shown in Figure 2. A threeway repeated measures ANOVA revealed that only the main effect of disparity was significant $(F(6,84)=22.28, p<.0001)$. The main effects of the flow direction (upward or downward) $(F(1,84)=1.19, p>.05)$ and the quality of the suppressor (static or dynamic dots) $(F(1,84)=.43, p>.05)$ were not significant. No interaction was significant $(p>.05)$. The results of the LSD tests were as the results from the duration data, except that the difference between 52.8 and 96.8 arcmin conditions was not significant here $(p>.05)$. 


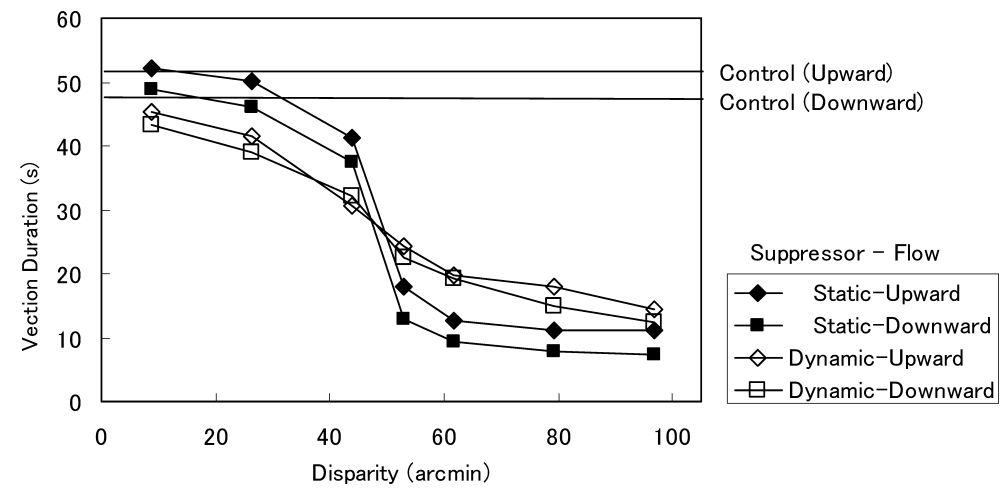

Fig. 2 Vection durations. Average vection durations in a trial of $60 \mathrm{~s}$. Horizontal lines in the figure indicate the results from the control conditions without the vection suppressor.

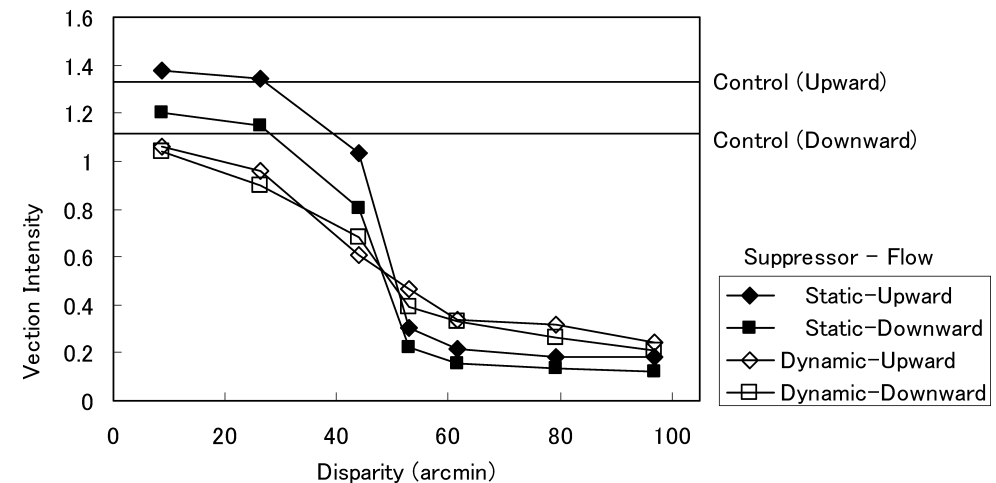

Fig. 3 Average vection intensity. Vection intensity was measured by the number of pushed buttons. The average intensity in a trial indicates 0 if no vection was reported through out one minute, and 2 if strong vection was reported during a whole trial. Horizontal lines in the figure indicate the results from the control conditions without the vection suppressor.

These statistics indicate that there is a gradual decrease in vection duration and strength according to the change in disparity of the suppressor from 26.4 to 52.8 arcmin, i.e., from a smaller disparity to the same disparity with that of the vection-inducing flow. The quantity of the suppression effect stayed the same within a range from 52.8 to $96.8 \mathrm{arcmin}$ disparity.

Asymmetry in the results between the upward and downward flow may exist. An upward flow may induce vection more strongly than a downward flow. However, such a difference was not revealed in the ANOVAs noted above. IF a large number of subjects had been used, it may have become apparent. However, the effect does not seem critical here, even if it existed.

\section{Discussion}

Our results show that the suppression effects of dynamic visual noise on vection have the same quality as those of a static visual pattern, especially when the dynamic noise is presented on a perceptually farther plane. This suggests that the vection suppression effect reported in the literature is not caused by the farther stimulus being stationary. The stationary or dynamic dots here should have dominated in producing vection when they were on a farther plane. However, they did not indicate a consistent flow direction in which vection was induced. We conclude that the vection suppression effect is explained simply by a dominance change from a consistent flow pattern inducing vection in one direction to an optical pattern inappropriate for inducing vection.

Applications using a virtual reality system make use of vection to emphasize the sense of presence. Amusement parks often utilize vection, presenting adventure-type movies on a huge screen (sometimes in 3-D). However, some people become sick when viewing such motion displays. This phenomenon is considered as a kind of motion sickness and called simulator sickness or cybersickness (McCauley and Sharkey, 1992). A widely accepted explanation for the phenomenon is that a conflict between signals from visual and vestibular systems causes it. Children seem to easily suffer from motion sickness. In Japan, there even was a case in which junior high school students were sent to the hospital because of motion sickness caused by a movie with jitter in 2003. Highly developed video-games (e.g., "Doom"), which show exciting image motion to simulate observer's views in a 3-D scene, cause simulator sickness even in adults. Regarding motion 
sickness, the recent popularity of large-screen TV sets or personal 3-D displays will make the environments worse especially for children.

Vection is a phenomenon in which visual signals indicating self-motion overcome vestibular signals indicating no selfmotion. Therefore, vection and simulator sickness must be closely related phenomena (Hettinger et al., 1990; Hettinger and Riccio, 1992; Lee et al., 1997; Smart et al., 2002). Although it is not clear whether vection is a cause of simulator sickness or not, it is possible that conditions that suppress (enhance) vection also suppress (enhance) motion sickness. In fact, jitter in movies seems to enhance vection (Palmisano et al., 2000), which would enhance motion sickness. It is also reported that a static scene seen through a head-mounted display impairs simulator sickness (Prothero et al., 1999), corresponding to the well-known phenomenon that the farther static stimulus suppresses vection.

The present study shows that dynamic visual noise impairs vection as effectively as static patterns do. It is possible that the dynamic stimulus also impairs motion sickness when it is presented on a depth plane farther than that of moving images. This means that dynamic noise may work as a vection (or motion sickness) suppressor used in movie applications. Dynamic visual noise may be more practical than static patterns, because static patterns sometimes break the whole movie construction whereas dynamic visual noise does little in this respect.

Another possibility is that dynamic visual noise suppresses vection but does not impair simulator sickness. This would suggest that vection itself is not a cause of simulator sickness. Dynamic visual noise does not indicate an objective vertical or absolute stability as a reference frame. If it could be shown that a reference frame impairs simulator sickness (Prothero et al., 1999), dynamic visual noise may not impair motion sickness, even though it impairs vection. If this is the case, it may be possible to search for an opposite condition where vection arises without motion sickness.

These possibilities should be examined in the future research. Such studies will contribute to producing safe and comfortable applications using moving images on a large screen without loss of the entertainment factor.

Acknowledgments This study was supported in part by Grant-in-Aid for the 21st Century COE Program entitled "Design of artificial environments on the basis of human sensitivity", Kyushu University, Japan. We thank Dr. Gerard Remijn for his comments on the manuscript.

\section{References}

Andersen GJ, Braunstein ML (1985) Induced self-motion in central vision. J Exp Psychol Hum Percept Perform 11: 122132

Hettinger LJ, Berbaum KS, Kennedy RS, Dunlap WP, Nolan MD (1990) Vection and simulator sickness. Mil Psychol 2:
171-181

Hettinger LT, Riccio GE (1992) Visually induced motion sickness in virtual environments. Presence 1: 306-310

Howard IP, Heckmann T (1989) Circular vection as a function of the relative sizes, distances and positions of two competing visual displays. Perception 18: 657-667

Ito H, Shibao T (1999) Motion direction distribution as a determinant of circular vection. Percept Mot Skills 89: 564570

Ito H, Fujimoto C (2003) Compound self-motion perception induced by two kinds of optical motion. Percept Psychophys 65: $874-887$

Ito $H$ (2004) Direction selectivity in visually induced selfmotion perception. Transactions of Virtual Reality Society of Japan 9: 35-40 [In Japanese]

Ito $\mathrm{H}$, Shibata I Self-motion perception from expanding and contracting optical flows overlapped with binocular disparity. Vision Res (in press)

Johansson G (1977) Studies on visual perception of locomotion. Perception 6: 365-376

Kano C (1991) The perception of self-motion induced by peripheral visual information in sitting and supine postures. Eco Psych 3: 241-252

Lee GCH, Yoo Y, Jones S (1997) Investigation of driving performance, vection, postural sway, and simulator sickness in a fixed-based driving simulator. Computers and Industrial Engineering 33: 533-536

Lo WT, So RHY (2001) Cybersickness in the presence of scene rotational movements along different axes. Appl Ergon 32: 1-14

MaCauley ME, Sharkey TJ (1992) Cybersickness: perception of self-motion in virtual environments. Presence 1: 311-318

Nakamura S, Shimojo S (1999) Critical role of foreground stimuli in perceiving visually induced self-motion (vection). Perception 28: 893-902.

Ohmi M, Howard IP, Landolt JP (1987) Circular vection as a function of foreground-background relationships. Perception 16: $17-22$

Ohmi M, Howard IP (1988) Effect of stationary objects on illusory forward self-motion induced by a looming display. Perception 17: 5-12

Palmisano S (1996) Perceiving self-motion in depth: The role of stereoscopic motion and changing-size cues. Percept Psychophys 58: 1168-1176

Palmisano S, Gillam BJ, Blackburn SG (2000) Globalperspective jitter improves vection in central vision. Perception 20: 57-67

Prothero J, Draper M, Furness T, Parker D, Wells M (1999) The use of an independent visual background to reduce simulator side-effects. Aviat Space Environ Med 70: 277283

Smart LJ, Stoffregen TA, Bardy BG (2002) Visually-induced motion sickness predicted by postural instability. Hum Factors 44: 451-465 
Received: September 6, 2004

Accepted: October 4, 2004

Correspondence to: Hiroyuki Ito, Department of Visual Communication Design, Kyushu University, 4-9-1 Shiobaru, Minami-ku, Fukuoka 815-8540, Japan

Phone: +81-92-553-4496

Fax: +81-92-553-4496

e-mail: ito@design.kyushu-u.ac.jp 